\title{
Lombardi Drawings of Graphs
}

\author{
Christian A. Duncan ${ }^{1}$, David Eppstein ${ }^{2}$, Michael T. Goodrich ${ }^{2}$, \\ Stephen G. Kobourov ${ }^{3}$, and Martin Nöllenburg ${ }^{2}$ \\ 1 Department of Computer Science, Louisiana Tech. Univ., Ruston, Louisiana, USA \\ 2 Department of Computer Science, University of California, Irvine, California, USA \\ 3 Department of Computer Science, University of Arizona, Tucson, Arizona, USA
}

\begin{abstract}
We introduce the notion of Lombardi graph drawings, named after the American abstract artist Mark Lombardi. In these drawings, edges are represented as circular arcs rather than as line segments or polylines, and the vertices have perfect angular resolution: the edges are equally spaced around each vertex. We describe algorithms for finding Lombardi drawings of regular graphs, graphs of bounded degeneracy, and certain families of planar graphs.
\end{abstract}

\section{Introduction}

The American artist Mark Lombardi [24] was famous for his drawings of social networks representing conspiracy theories. Lombardi used curved arcs to represent edges, leading to a strong aesthetic quality and high readability. Inspired by this work, we introduce the notion of a Lombardi drawing of a graph, in which edges are drawn as circular arcs with perfect angular resolution: consecutive edges are evenly spaced around each vertex. While not all vertices have perfect angular resolution in Lombardi's work, the even spacing of edges around vertices is clearly one of his aesthetic criteria; see Fig. 1 .

Traditional graph drawing methods rarely guarantee perfect angular resolution, but poor edge distribution can nevertheless lead to unreadable drawings. Additionally, while some tools provide options to draw edges as curves, most rely on straight-line edges, and it is known that maintaining good angular resolution can result in exponential drawing area for straight-line drawings of planar graphs [1725]. Our requirement of perfect angular resolution forces us to use curved edges, since even very simple graphs such as cycle graphs cannot be drawn with perfect angular resolution and straight edges.

New Results. We define a Lombardi drawing of a graph $G$ to be a drawing of $G$ in the plane in which vertices are represented as points (or as disks or labels centered on those points), edges are represented as line segments or circular arcs between their endpoints, and every vertex has perfect angular resolution, as measured by the angle formed by the tangents to the edges at the vertex. We do not necessarily insist that the drawings are free of crossings; the drawings of Lombardi had crossings, sometimes even in cases where they could have been avoided. We also do not consider crossings when we measure the angular resolution of a drawing. However, we do require that the only vertices that intersect the arc for an edge $(u, v)$ are its two endpoints $u$ and $v$.

Several of Mark Lombardi's drawings used a circle as their overall shape. We define a circular Lombardi drawing to be a Lombardi drawing in which the vertices lie on a

U. Brandes and S. Cornelsen (Eds.): GD 2010, LNCS 6502, pp. 195207, 2011.

(C) Springer-Verlag Berlin Heidelberg 2011 


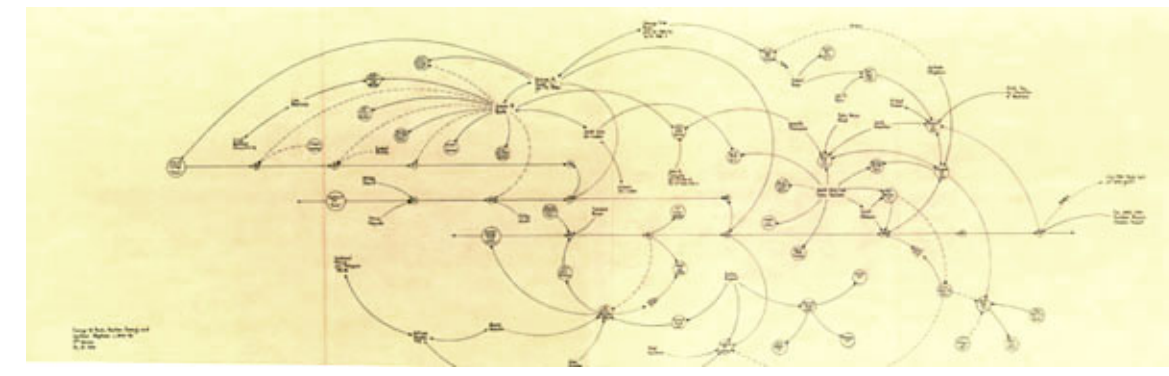

Fig. 1. Mark Lombardi, George W. Bush, Harken Energy, and Jackson Stevens c.1979-90, 1999. Graphite on paper, $20 \times 44$ inches [24].

circle. Similarly, we define a $k$-circular Lombardi drawing to be a Lombardi drawing in which the vertices lie on $k$ concentric circles. We provide the following:

- We characterize the regular graphs that have circular Lombardi drawings, and we find efficient algorithms for constructing these drawings.

- We describe methods of finding Lombardi drawings for any 2-degenerate graph (a graph that may be reduced to the empty graph by repeated removal of vertices of degree at most 2) and many but not all 3-degenerate graphs.

- We investigate the graphs that have planar Lombardi drawings. We show that certain subclasses of the planar graphs always have such drawings, but that there exist planar graphs with no planar Lombardi drawing.

- We implement an algorithm for constructing $k$-circular Lombardi drawings and use it to draw many symmetric graphs.

Related Work. Although most previous work on angular resolution concerns straightline drawings (e.g., see [10|17|25]) or polyline drawings (e.g., see [18|21]), the angular resolution of drawings with circular-arc edges was previously studied by Cheng et al. [8], who showed that maintaining bounded angular resolution in planar drawings may require exponential area even with circular-arc edges. Our circular Lombardi drawings use a circular layout of vertices that is already popular (e.g., see [3|16|30]). However, previous methods for circular layouts draw edges as straight line segments or curves perpendicular to the circle, neither of which leads to good angular resolution.

Efrat $e t$ al. [13] show that given a fixed placement of the vertices of a planar graph, determining whether the edges can be drawn with circular arcs so that there are no crossings is NP-Complete. For fixed position drawings with cubic Bézier curves, Brandes et al. [5]7] use force-directed algorithms to maximize the angular resolution and Brandes, Shubina, and Tamassia [6] rotate optimal angular resolution templates. Aicholzer et al. [1] show that, for a given embedded planar triangulation with fixed vertex positions, one can find a circular-arc drawing of the triangulation that maximizes the minimum angular resolution by solving a linear program. Finkel and Tamassia [14] also try to optimize angular resolution using force-directed methods for laying out graphs with curved edges. Di Battista and Vismara [10] give a nonlinear optimization characterization that can find straight-line drawings of embedded planar graphs with a prescribed assignment of angles if such drawings exist. 
Any tree may be drawn with straight edges and perfect angular resolution. However, in a separate paper [12], we show that (when the order of the edges is fixed around each vertex) straight-line tree drawings with perfect angular resolution may require exponential area, whereas Lombardi drawings can achieve polynomial area.

\section{Circular Lombardi Drawings of Regular Graphs}

We begin by investigating circular Lombardi drawings, Lombardi drawings in which all vertices are placed on a circle. As we show, drawings of this type exist for many regular graphs. Our proofs use the following basic geometric observation:

Property 1. Let $A$ be a circular arc or line segment connecting two points $p$ and $q$ that both lie on circle $O$. Then $A$ makes the same angle to $O$ at $p$ that it makes at $q$. Moreover, for any $p$ and $q$ on $O$ and any angle $0 \leq \theta \leq \pi$, there exists an arc, line segment, or pair of collinear rays $A$ connecting $p$ and $q$, making angle $\theta$ with $O$, and lying either inside or outside of $O$.

The case of two collinear rays is problematic (we only allow edges to be represented by arcs or line segments) but easily avoided by perturbing the vertices on $O$.

Lemma 1. A $d$-regular graph $G$ has a circular Lombardi drawing if and only if $G$ can be decomposed into a disjoint union of 1-regular and 2-regular graphs and one of the following conditions is true: $d \not \equiv 2(\bmod 4)$, one of the 2-regular subgraphs is bipartite, or one of the 2-regular subgraphs is a Hamiltonian cycle.

Proof. Suppose $G$ has a circular Lombardi drawing on a circle $O$ centered at $o$; in this drawing, define the twist $\theta_{v}$ of a vertex $v$ to be the sharpest of the angles between line segment $v o$ and the edges incident to $v$ (with positive sign if one of the edges forming the sharpest angle is clockwise of the line segment, and negative sign if there is only one edge forming the sharpest angle and it is counterclockwise of $v$ ). Then if $v$ and $w$ are adjacent in $G, \theta_{v}=-\theta_{w}$ except when there are two equal sharpest angles at both $v$ and $w$, in which case $\theta_{v}=\theta_{w}$. In each connected component either all vertices have the same twist, and have edge angles that are symmetric with respect to reflections through axis vo, or the component is bipartite, all vertices on one side of the bipartition have one twist, and all vertices on the other side of the bipartition have the opposite twist.

We can decompose each connected component of $G$ into 1-regular and 2-regular graphs by partitioning the edges of the component according to the angle they make with circle $O$. For a bipartite component in which the vertices on the two sides of the bipartition have different twists, this forms a decomposition into 1-regular graphs (some of which may be combined in pairs to form bipartite 2-regular graphs). When $d$ is 2 mod 4 and a component of $G$ is not bipartite, the only possibilities for a symmetric twist are to make some edges parallel or perpendicular to $O$. Edges that are parallel to $O$ must be drawn as arcs of $O$ through all vertices, so they form a Hamiltonian cycle. Edges perpendicular to $O$ must form even-length cycles that alternate between the inside and outside of $O$. Thus, in all cases a graph with a circular Lombardi drawing can be decomposed into 1-regular and 2-regular graphs matching the conditions of the lemma. 
In the other direction, suppose that $G$ can be decomposed into 1-regular and 2-regular graphs with the additional conditions of the lemma. By combining pairs of 1-regular graphs into a single 2-regular graph, we may assume that all but at most one of these subgraphs are 2-regular. Then we may choose an evenly spaced set of angles, draw each 2 -regular graph as a set of arcs that meet $O$ at one of these fixed angles, and draw the 1-regular graph (if it exists) as a set of arcs that are perpendicular to and interior to $O$. If $d$ is divisible by four, we can choose these angles in such a way that no angle is parallel to the circle $O$ and no angle is perpendicular to $O$. If $d$ is odd, the angles can be chosen so that the 1-regular subgraph of $G$ is perpendicular to and interior to $O$, and all other angles are neither perpendicular nor parallel to $O$. If $d$ is congruent to 2 mod 4 and one of the 2-regular graphs is a Hamiltonian cycle, we may draw it using edges that lie on $C$, placing the vertices in the order of this cycle. And if $d$ is congruent to $2 \bmod 4$ and one of the 2-regular graphs is bipartite, we may draw it using edges that are perpendicular to $O$, taking care in the vertex placement to avoid using an edge that connects two diametrally opposite points on $O$ via an exterior arc. In both of these cases where $d$ is $2 \bmod 4$ we then draw the other subgraphs of the decomposition using arcs that are neither parallel to nor perpendicular to $O$.

Theorem 1. Every regular graph $G$ of degree divisible by four has a circular Lombardi drawing. A regular graph of odd degree has a circular Lombardi drawing if and only if it has a perfect matching. A regular graph of degree congruent to two modulo four has a circular Lombardi drawing if and only if it is Hamiltonian or has a 2-regular bipartite subgraph. In the cases of odd degree and degree divisible by four, when a circular Lombardi drawing exists it can be constructed in polynomial time.

Proof. This follows from Lemma 1 together with Petersen's theorem that a regular graph of even degree can always be decomposed into 2-regular subgraphs [27|28].

Testing for the existence of a 2-regular bipartite subgraph in a regular graph is NPcomplete (in 3-regular graphs, it is equivalent to 3-edge-coloring) but we have not determined its complexity for the case of interest to us, $d$-regular graphs in which $d$ is congruent to two modulo four.

Figures 2(a-c) show drawings produced by this method for 3-regular, 4-regular, and 6-regular graphs. Figure 2(d) shows a 3-regular graph that does not have a perfect matching, and that therefore has no circular Lombardi drawing.

For bipartite regular graphs of bounded degree the method of Theorem 1 again leads to a linear-time algorithm.

Corollary 1. Every bipartite d-regular graph has a circular Lombardi drawing that can be constructed in time $O(d n \log d)$.

Proof. It is known that every bipartite regular graph can be decomposed into perfect matchings in the given time bound [2 9229$]$. 1 The result follows by applying Theorem 1 to this decomposition.

\footnotetext{
${ }^{1}$ The fact that every regular bipartite graph has a decomposition into matchings is commonly attributed to König [22], but is equivalent to a result proved in terms of point-line configurations in the $1894 \mathrm{Ph} . \mathrm{D}$. thesis of Ernst Steinitz.
} 


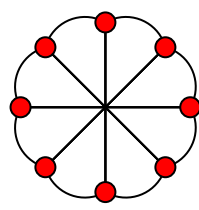

(a)

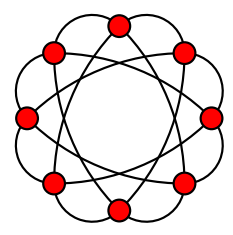

(b)

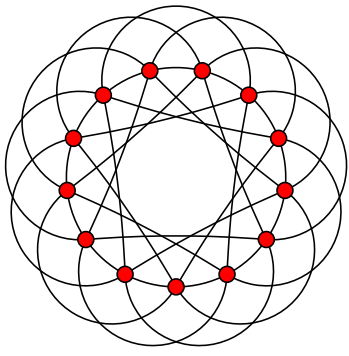

(c)

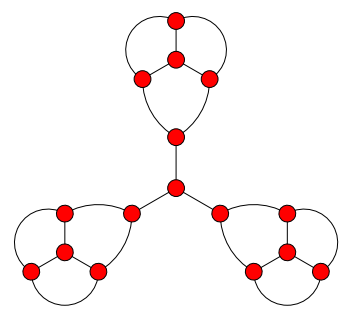

(d)

Fig. 2. (a) A circular Lombardi drawing of the 3-regular Wagner graph; (b) A circular Lombardi drawing of the 4-regular graph $K_{4,4}$; (c) The 6-regular Paley graph connecting integers modulo 13 if their difference is a quadratic residue; (d) A 3-regular graph that has no perfect matching and therefore has no circular Lombardi drawing

Corollary 2. Every d-regular graph for which $d$ is a power of two, with the exception of 2-regular non-bipartite disconnected graphs, has a circular Lombardi drawing that can be constructed in time $O(d n \log d)$.

Proof. Repeatedly decompose the graph into pairs of subgraphs with half the degree by taking alternating edges of an Euler tour [15] and then apply Theorem 1 to the decomposition.

Corollary 3. Every 3-regular bridgeless graph has a circular Lombardi drawing that can be constructed in time $O\left(n \log ^{3} n \log \log n\right)$.

Proof. The result that every 3-regular bridgeless graph has a perfect matching (equivalently, a decomposition into a 2-regular and a 1-regular subgraph) is known as Petersen's theorem [28]. Such a matching can be found in the stated time bound via an algorithm based on dynamic 2-edge-connectivity testing data structures [4]2031].

\section{Two-Degenerate and Three-Degenerate Graphs}

The degeneracy of a graph $G$ is the minimum number $d$ such that $G$ can be reduced to the empty graph by repeatedly removing a vertex of degree at most $d$; equivalently, it is the minimum degree in the subgraph of $G$ that maximizes the minimum degree [23]. If a graph $G$ has degeneracy at most $d$, it is known as $d$-degenerate. In this section we consider algorithms for drawing 2-degenerate and 3-degenerate graphs, with a specified cyclic ordering of the edges around each vertex. The main idea of these algorithms is to delete a low-degree vertex, draw the remaining graph with the appropriate angles at each of its vertices, and then find a position for the deleted vertex that allows it to be connected to the drawing of the remaining graph.

For 2-degenerate graphs, when we add back the vertices in reverse order of deletion, there is always a circle on which they can be added so we can choose one point on the 
circle that is not crossed by a previously drawn feature. For 3-degenerate graphs there are two points at which the point can be added to give the correct edge angles (the common intersection points of three circles) so there might be circumstances under which this addition is forced to create an undesirable edge-vertex or vertex-vertex intersection.

The results in this section rely on the following geometric property, which is proven in the full version of the paper [11].

Property 2. Suppose we are given two points $p$ and $q$ with associated vectors $v_{p}$ and $v_{q}$ and an angle $\theta_{p q}$. Consider all pairs of circular arcs that leave $p$ and $q$ with tangent vectors $v_{p}$ and $v_{q}$ respectively and meet at an angle $\theta_{p q}$. The locus of meeting points for these pairs of arcs is a circle.

\subsection{2-Degenerate Graphs}

Theorem 2. Every 2-degenerate graph with a specified cyclic ordering of the edges around each vertex has a Lombardi drawing.

Proof. Order the vertices by repeatedly removing a low-degree vertex. Reinsert the vertices in reverse order creating subgraphs $G_{0}, G_{1} \ldots G_{n}$ with the invariant that after each insertion the drawing is a partial Lombardi drawing $\Gamma_{i}$ of $G_{i}$ where some vertices may not yet have all of their neighbors placed. To insert a new vertex $v=v_{i+1}$ with degree two in $G_{i+1}$ (the case for degree one is simpler) let $p$ and $q$ be its two neighbors in $G_{i+1}$. Since there is a specified ordering around $p$, which has already been placed in $\Gamma_{i}$, there is a unique tangent vector $v_{p}$ associated with the arc from $p$ to $v$. Similarly, there is a unique tangent vector $v_{q}$. In addition, since the degree of $v$ in $G$ is known and the ordering of the neighbors at $v$ is also given, there is a unique angle $\theta_{p q}$ associated with the two arcs from $p$ and $q$ to $v$. From Property 2, we may choose to place $v$ at any position on the defined circle. Choosing a point $v$ that does not coincide with any other arcs or vertices already placed guarantees we have a valid drawing $\Gamma_{i+1}$.

Corollary 4. Every outerplanar or series-parallel graph has a Lombardi drawing.

Proof. This follows from the fact that these graphs are 2-degenerate.

\subsection{3-Degenerate Graphs}

An algorithm following the same approach can be used to draw many, but not all, 3degenerate graphs. In this case we have three points $p, q$, and $r$ that we want to connect by arcs to an unplaced new vertex $v$. Each pair of known points yields a circle of possible choices for $v$. These three circles, $O_{p q}, O_{p r}, O_{q r}$, have to pairwise cross, and where they cross the third one must also cross because fixing the angles between two pairs of incoming arcs at the new point fixes all angles. Every graph with maximum degree four is either 4-regular or 3-degenerate, so the same algorithm applies in this case.

However, for certain graphs and certain orderings of the edges around the vertices of the graph, this algorithm can fail by placing a vertex on another edge or vertex. An example in which this occurs is the seven-vertex split graph $G_{7}$ formed by adding four 


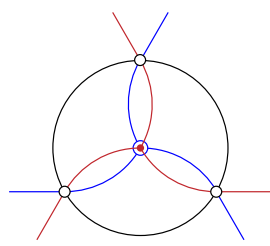

(a)

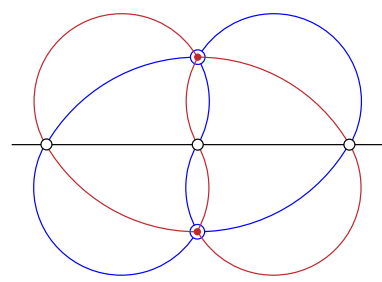

(b)

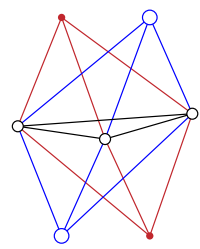

(c)

Fig. 3. A 7-vertex 3-degenerate graph that has no Lombardi drawing with the given vertex ordering. (a) A Möbius transformation makes one triangle equilateral, forcing the other 4 vertices to be placed at the centroid and the point at infinity; (b) A different transformation with finite vertex locations; (c) A straight-line drawing of the graph.

independent vertices $p, q, r$, and $s$ to a triangle $x y z$, with an edge from each of $p, q, r$, and $s$ to each of $x, y$, and $z$, as shown in Figure 3 In any Lombardi drawing of $G_{7}$ with the edge order as shown, we can assume by making an appropriate Möbius transformation of the drawing that $x y z$ is equilateral. It follows that the only possible locations for $p$, $q, r$, and $s$ are the centroid of the equilateral triangle and the point at infinity, so at least two vertices would have to be placed at the same point, forming an invalid drawing.

\section{Non-crossing Lombardi Drawings}

\subsection{Planar Graphs without Planar Lombardi Drawings}

Not every planar graph has a planar Lombardi drawing. To see this, consider the $k$ nested triangle graphs, maximal planar graphs with $3 k$ vertices formed by $k$ nested triangles with $k-1$ six-cycles connecting consecutive triangles. A $k$-nested triangle graph may also be formed geometrically by gluing $k-1$ octahedra end-to-end.

As can be seen in Figure 4 the 2-nested and 3-nested triangle graphs have planar Lombardi drawings. The 4-nested triangle graph, however, does not. If it did have such a drawing, its middle two triangles would form circles (the only smooth curve formed by three circular arcs). By an appropriate Möbius transformation, the outer circle $O$ can be assumed to have its three vertices equally spaced around it. The three cirles $C_{1}, C_{2}$, and $C_{3}$ that (by Property 2) describe the potential positions of the vertices on the inner circle have the same radius as $O$ and meet at the center of $O$, and the inner circle would have to be tangent to all three of $C_{1}, C_{2}$, and $C_{3}$. However, the only circle tangent to all three is exterior to $O$, concentric with $O$ and having twice the radius of $O$. Therefore, using an edge ordering around each vertex that comes from a planar embedding but enforcing perfect angular resolution leads to a nonplanar drawing, shown in Figure 4(c).

\subsection{Halin Graphs}

A Halin graph [19] is a planar graph obtained from a plane tree $T$ (with at least four vertices and with no vertices of degree 2), by connecting all the leaves of $T$ into a 


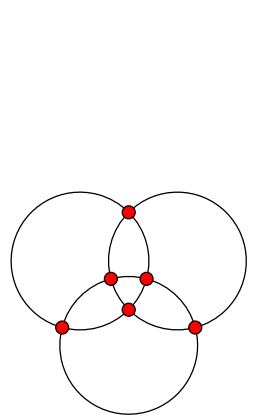

(a) $k=2$

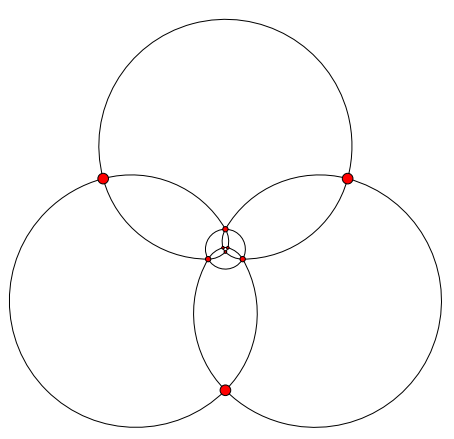

(b) $k=3$

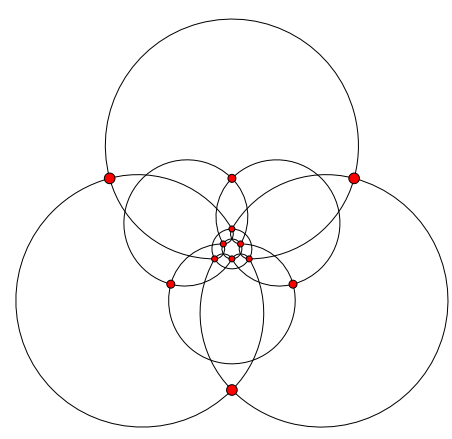

(c) $k=4$

Fig. 4. $k$-nested triangle graphs. The 2-nested and 3-nested triangle graphs have planar Lombardi drawings, but the 4-nested triangle graph does not.

cycle in the order given by its embedding. As we now describe, Halin graphs (and the graphs formed in the same way from trees with degree-2 vertices) have planar Lombardi drawings that can be constructed using hyperbolic geometry.

We draw $T$ within a Poincaré disk model of the hyperbolic plane, with its leaves on the boundary circle of the model, and then draw the cycle connecting the leaves outside this circle. If $T$ is drawn using hyperbolic line segments, with perfect angular resolution, then its edges will form circular arcs in the Poincare model; the conformal (angle-preserving) nature of the Poincare model implies that the angular resolution of the hyperbolic line segments equals the angular resolution of these Euclidean arcs.

For a given straight-line drawing of a rooted tree in the hyperbolic plane, and a nonroot vertex $v$, partition the hyperbolic plane into wedges bounded by the bisectors of the angles around the parent of $v$ and define the dominance region of $v$ to be the wedge containing $v$. Equivalently, in a Voronoi diagram generated by the rays from the parent of $v$ to its children, the dominance region of $v$ is the Voronoi cell containing $v$. We define a good hyperbolic drawing of a rooted tree $T$ to be a drawing in which the edges are straight line segments or rays in the hyperbolic plane, the leaves are placed on the circle at infinity, and the dominance regions for two vertices $v$ and $w$ are either nested within each other (if one of the two vertices is an ancestor of the other) or disjoint otherwise. Two dominance regions in a good hyperbolic drawing are shown in Figure 5 a).

Lemma 2. Every rooted tree has a good hyperbolic drawing.

Proof. We use induction on the number of non-leaf nodes in the given tree $T$. As a base case, when there is one non-leaf node, it may be placed at the center of the Poincaré disk model of the hyperbolic plane with its leaves at the limit points of equally-spaced rays (radii of the disk model). Otherwise, let $v$ be a non-leaf that is as far from the root of $T$ as possible, and let $T^{\prime}$ be formed from $T$ by removing all children of $v$. Then by induction, $T^{\prime}$ has a good hyperbolic drawing. In this drawing, $v$ is on the circle at infinity; let $R$ be the ray connecting the parent of $v$ to $v$. For any position $x$ along this ray, let $\theta_{x}$ be the maximum angle made to $R$ by a line that stays within the dominance 


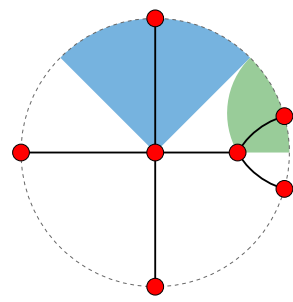

(a)

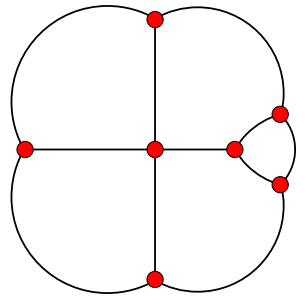

(b)

Fig. 5. (a) A good hyperbolic drawing of a seven-node tree, with the dominance regions of two leaves of the tree shown as shaded regions; (b) The Lombardi drawing formed by adding arcs outside the Poincaré model, at $30^{\circ}$ angles to the boundary, connecting consecutive leaves

region of $v$. Then $\theta_{x}$ varies continuously along $R$, starting from a value of $\pi / d$ at the parent of $v$ (where $d$ is the degree of the parent) and ending with a value of $\pi$ at $v$ itself. If the degree of $v$ in $T$ is $d^{\prime}$, there must be an intermediate position $x$ on $R$ for which $\theta_{x}=\pi\left(1-1 / d^{\prime}\right)$. If we move $v$ to $x$ and place its leaf children at the limit points of equally spaced rays around $x$, the result is a good hyperbolic drawing of $T$.

Theorem 3. Every Halin graph has a planar Lombardi drawing that may be constructed in linear time.

Proof. Root the tree $T$ at an arbitrarily chosen non-leaf node, and construct a good hyperbolic drawing of $T$ according to Lemma 2 Draw the cycle connecting the leaves of $T$ using circular arcs that meet the circle bounding the Poincaré model at angles of $30^{\circ}$ as in Figure 5 (b). Then each non-leaf node of $T$ has perfect angular resolution from the tree drawing, and each leaf node has perfect angular resolution because the ray connecting it to its parent in $T$ is perpendicular to the boundary circle and therefore at $120^{\circ}$ angles from the two arcs connecting it to adjacent leaves.

\subsection{Other Classes of Planar Graphs}

The networks formed by two-dimensional soap bubbles naturally form 3-regular planar Lombardi drawings: they have circular arcs as their edges (the boundaries between bubbles), and $120^{\circ}$ angles at each vertex where three arcs meet [26]. However, we do not have a precise characterization of the graphs that can be formed in this way.

The vertices of every Platonic solid, Archimedean solid, and prism lie on a common sphere. In all but two cases (the snub cube and snub dodecahedron) one may draw the edges of the polyhedron as circular arcs on the sphere with perfect angular resolution. By stereographic projection, each of these graphs has a Lombardi drawing in the plane. For instance, Figure 4(a) depicts the graph of the octahedron drawn in this way.

All outerplanar and series-parallel graphs have Lombardi drawings (Corollary 4), but we do not know whether they all have planar Lombardi drawings. 


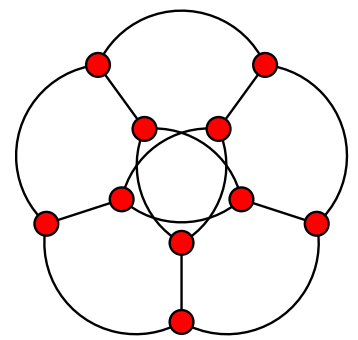

(a) Petersen graph

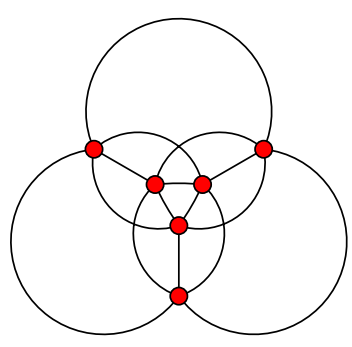

(b) $K_{6}$

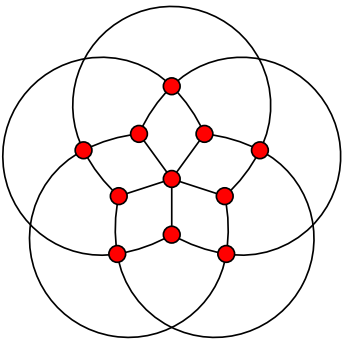

(c) Grötzsch graph

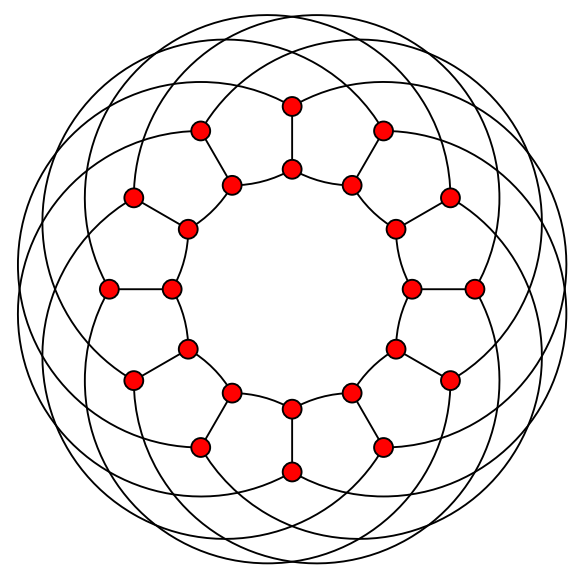

(d) Nauru graph $G(12,5)$

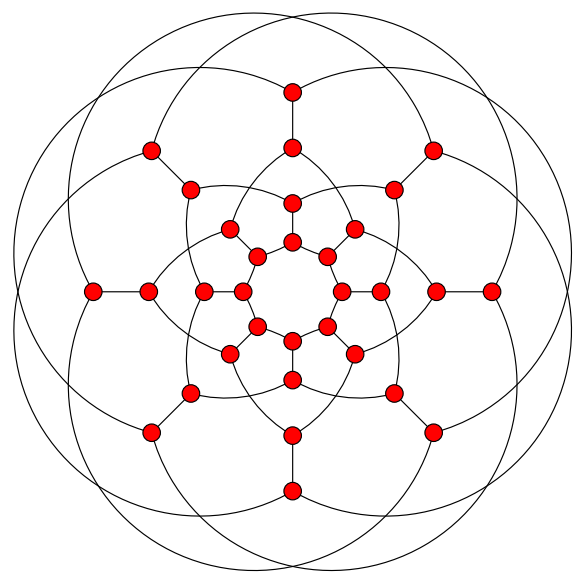

(f) Dyck graph

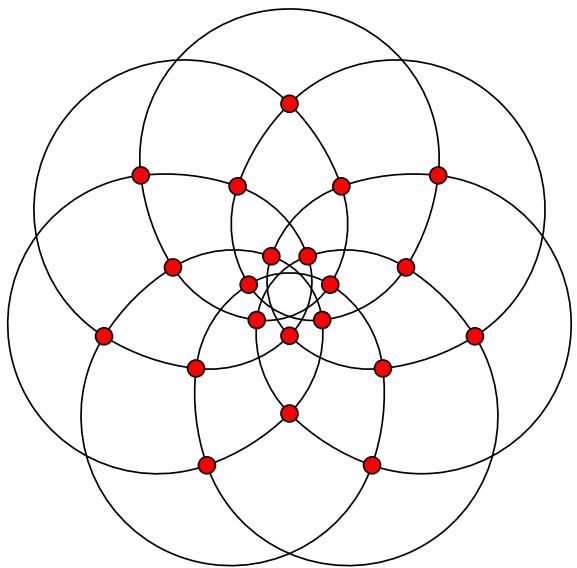

(e) Brinkmann graph

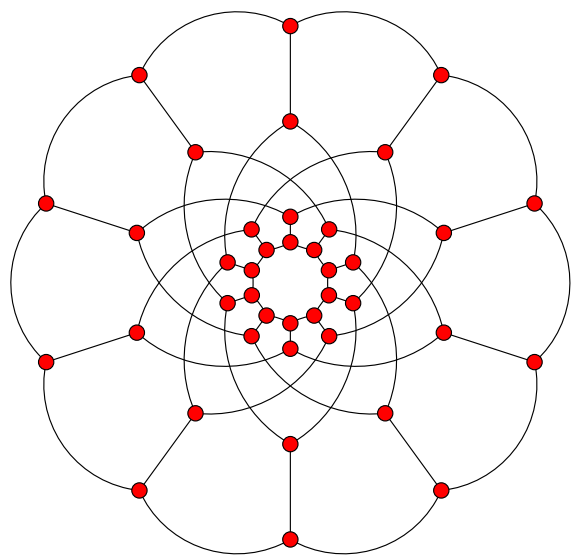

(g) 40-vertex cubic symmetric graph $F_{40}$ (the bipartite double cover of the dodecahedron)

Fig. 6. Sample drawings by the Lombardi Spirograph 


\section{The Lombardi Spirograph}

We have implemented a program for constructing $k$-circular Lombardi drawings of graphs with dihedral symmetry; we call it the Lombardi Spirograph, as its drawings resemble those created by the Spirograph ${ }^{\mathrm{TM}}$ drawing toy produced by Hasbro, Inc. Our program places vertices on $k$ concentric circles; the input specifies not only the number of vertices per circle and the set of edges to be drawn, but also the order in which those edges are incident at each vertex. Each vertex can have at most three neighbors on smaller circles; a circle on which the vertices have two or three inward neighbors has a unique radius for which the vertices have perfect angular resolution, whereas the radius for circles on which the vertices have one inner neighbor is chosen heuristically.

Figures $2(\mathrm{a}-\mathrm{c}), 4(\mathrm{a} \& \mathrm{~b})$, and 6 were all drawn using this program.

\section{Conclusions}

We have begun an investigation into Lombardi drawings and found algorithms based on graph matching, incremental construction, hyperbolic geometry, and symmetry display for constructing drawings of this type. Based on our constructions, we can show that many regular graphs, sparse graphs, special classes of planar graphs, and symmetric graphs have Lombardi drawings, and we have found drawings of this type for many well-known graphs. In addition, we have implemented a method, called the Lombardi Spirograph, for producing Lombardi drawings of graphs with dihedral symmetry.

There are many related problems that remain open, including the following:

1. What are the complexities of finding circular Lombardi drawings for regular graphs with degrees that are $2 \bmod 4$ ?

2. Is there an effective classification of 3-degenerate graphs according to whether they can or cannot be drawn in a way that avoids overlapping features?

3. Are there efficient methods for producing planar Lombardi drawings for outerplanar graphs, series-parallel graphs, and 3-regular planar graphs?

It would also be of interest to combine Lombardi drawing with other standard graph drawing quality criteria such as edge-length minimization. In general, we believe that Lombardi drawings will be a fruitful area for much additional research.

\section{Acknowledgments}

This research was supported in part by the National Science Foundation under grant 0830403, by the Office of Naval Research under MURI grant N00014-08-1-1015, and by the German Research Foundation under grant NO 899/1-1.

\section{References}

1. Aichholzer, O., Aigner, W., Aurenhammer, F., Dobiášová, K.Č., Jüttler, B.: Arc triangulations. In: Proc. 26th Eur. Worksh. Comp. Geometry (EuroCG 2010), Dortmund, Germany, pp. 17-20 (2010) 
2. Alon, N.: A simple algorithm for edge-coloring bipartite multigraphs. Information Processing Letters 85(6), 301-302 (2003), doi:10.1016/S0020-0190(02)00446-5

3. Baur, M., Brandes, U.: Crossing reduction in circular layouts. In: Hromkovič, J., Nagl, M., Westfechtel, B. (eds.) WG 2004. LNCS, vol. 3353, pp. 332-343. Springer, Heidelberg (2004), http://www.springerlink.com/content/fepu2a3hd195ffjg/

4. Biedl, T.C., Bose, P., Demaine, E.D., Lubiw, A.: Efficient algorithms for Petersen's matching theorem. J. Algorithms 38(1), 110 (2001), doi:10.1006/jagm.2000.1132

5. Brandes, U., Schlieper, B.: Angle and distance constraints on tree drawings. In: Kaufmann, M., Wagner, D. (eds.) GD 2006. LNCS, vol. 4372, pp. 54-65. Springer, Heidelberg (2007), doi:10.1007/978-3-540-70904-6_7

6. Brandes, U., Shubina, G., Tamassia, R.: Improving angular resolution in visualizations of geographic networks. In: Data Visualization 2000. Proc. 2nd Eurographics/IEEE TCVG Symp. Visualization (VisSym 2000), pp. 23-32. Springer, Heidelberg (2000)

7. Brandes, U., Wagner, D.: Using graph layout to visualize train interconnection data. J. Graph Algorithms Appl. 4(3), 135-155 (2000)

8. Cheng, C.C., Duncan, C.A., Goodrich, M.T., Kobourov, S.G.: Drawing planar graphs with circular arcs. Discrete Comput. Geom. 25(3), 405-418 (2001), doi:10.1007/s004540010080

9. Cole, R., Ost, K., Schirra, S.: Edge-coloring bipartite multigraphs in $O(E \log D)$ time. Combinatorica 21(1), 5-12 (2001), doi:10.1007/s004930170002

10. di Battista, G., Vismara, L.: Angles of planar triangular graphs. SIAM J. Discrete Math. 9(3), 349 (1996), doi:10.1137/S0895480194264010

11. Duncan, C.A., Eppstein, D., Goodrich, M.T., Kobourov, S.G., Nöllenburg, M.: Lombardi Drawings of Graphs. (September 2010) ArXiv e-prints, arXiv:1009.0579

12. Duncan, C.A., Eppstein, D., Goodrich, M.T., Kobourov, S.G., Nöllenburg, M.: Drawing trees with perfect angular resolution and polynomial area. In: Proc. 18th Int. Symp. on Graph Drawing (GD 2010), Springer, Heidelberg (2010)

13. Efrat, A., Erten, C., Kobourov, S.G.: Fixed-location circular arc drawing of planar graphs. J. Graph Algorithms Appl. 11(1), 145-164 (2007), http://jgaa.info/accepted/2007/EfratErtenKobourov2007.11.1.pdf

14. Finkel, B., Tamassia, R.: Curvilinear graph drawing using the force-directed method. In: Pach, J. (ed.) GD 2004. LNCS, vol. 3383, pp. 448-453. Springer, Heidelberg (2005), doi:10.1007/978-3-540-31843-9_46

15. Gabow, H.N.: Using Euler partitions to edge color bipartite multigraphs. Int. J. Parallel Programming 5(4), 345-355 (1976), doi:10.1007/BF00998632

16. Gansner, E.R., Koren, Y.: Improved circular layouts. In: Kaufmann, M., Wagner, D. (eds.) GD 2006. LNCS, vol. 4372, pp. 386-398. Springer, Heidelberg (2007), doi:10.1007/978-3540-70904-6_37

17. Garg, A., Tamassia, R.: Planar drawings and angular resolution: algorithms and bounds. In: van Leeuwen, J. (ed.) ESA 1994. LNCS, vol. 855, pp. 12-23. Springer, Heidelberg (1994), doi:10.1007/BFb0049393

18. Gutwenger, C., Mutzel, P.: Planar polyline drawings with good angular resolution. In: Whitesides, S.H. (ed.) GD 1998. LNCS, vol. 1547, pp. 167-182. Springer, Heidelberg (1999), doi:10.1007/3-540-37623-2_13

19. Halin, R.: Über simpliziale Zerfällungen beliebiger (endlicher oder unendlicher) Graphen. Math. Ann. 156(3), 216-225 (1964), doi:10.1007/BF01363288

20. Holm, J., de Lichtenberg, K., Thorup, M.: Poly-logarithmic deterministic fully-dynamic algorithms for connectivity, minimum spanning tree, 2-edge, and biconnectivity. J. ACM 48(4), 723-760 (2001), doi:10.1145/502090.502095

21. Kant, G.: Drawing planar graphs using the canonical ordering. Algorithmica 16, 4-32 (1996), doi:10.1007/BF02086606 
22. Kőnig, D.: Gráfok és mátrixok. Matematikai és Fizikai Lapok 38, 116-119 (1931)

23. Lick, D.R., White, A.T.: K-degenerate graphs. Canad. J. Math. 22, 1082-1096 (1970), http://www.smc.math.ca/cjm/v22/p1082

24. Lombardi, M., Hobbs, R.: Mark Lombardi: Global Networks. Independent Curators (2003)

25. Malitz, S., Papakostas, A.: On the angular resolution of planar graphs. SIAM J. Discrete Math. 7(2), 172-183 (1994), doi:10.1137/S0895480193242931

26. Morgan, F.: Soap bubbles in $\mathbb{R}^{2}$ and in surfaces. Pacific J. Math. 165(2), 347-361 (1994), http://projecteuclid.org/euclid.pjm/1102621620

27. Mulder, H.M.: Julius Petersen's theory of regular graphs. Discrete Mathematics 100(1-3), 157-175 (1992), doi:10.1016/0012-365X(92)90639-W

28. Petersen, J.: Die Theorie der regulären Graphs. Acta Math. 15(1), 193-220 (1891), doi:10.1007/BF02392606

29. Schrijver, A.: Bipartite edge coloring in $O(\Delta m)$ time. SIAM J. Comput. 28(3), 841-846 (1999), doi:10.1137/S0097539796299266

30. Six, J.M., Tollis, I.G.: A framework for circular drawings of networks. In: Kratochvíl, J. (ed.) GD 1999. LNCS, vol. 1731, pp. 107-116. Springer, Heidelberg (1999)

31. Thorup, M.: Near-optimal fully-dynamic graph connectivity. In: Proc. 32nd ACM Symp. Theory of Computing (STOC 2000), pp. 343-350 (2000), doi:10.1145/335305.335345 